\title{
Dry eye disease
}

\author{
Rahul A. Sharma BSc, Rookaya Mather MD
}

\section{Dry eye disease may substantially affect quality of life}

Dry eye disease refers to a group of disorders that involve reduced tear production or excessive tear evaporation. ${ }^{1}$ Dry eye affects up to $30 \%$ of people older than 50 years of age. ${ }^{2}$ Studies evaluating the effect of moderate and severe dry eye on quality of life have shown utility scores similar to those for patients with moderate to severe angina or those receiving dialysis. $^{3}$

Artificial tears are first-line therapy for mild dry eye disease

Symptomatic improvement with the use of artificial tears four times per day (first-line therapy) should occur within days, but it may take up to three to four weeks for some patients. ${ }^{1}$ When appropriate, environmental strategies (e.g., frequent blinking, avoidance of air currents, use of a humidifier) may be helpful. The patient's medication use should be reviewed because medications that may exacerbate dry eye (e.g., diuretics, selective serotonin receptor inhibitors, tricyclic antidepressants, $\beta$-blockers) may need adjustment. Antihistamine medications and local agents that promote vasoconstriction should be avoided. ${ }^{1}$

CMAJ invites submissions to "Five things to know about ..." Submit manuscripts online at http://mc.manuscriptcentral .com/cmaj

\section{The diagnosis of dry eye is based on clinical presentation}

Patients with mild dry eye disease may have conjunctival injection, as well as irritation, itching, soreness, burning or intermittent blurred vision. Visual deterioration may occur in severe cases secondary to ocular surface desiccation or keratinization, or may be a result of corneal scarring, thinning or ulceration. ${ }^{1}$ Provided that other causes of red eye (e.g., conjunctivitis, blepharitis, contact lens-related keratitis) can be excluded, therapy can be initiated based on signs and symptoms alone.,

\section{Excessive use of preserved artificial tears can exacerbate the symptoms of dry eye}

The preservatives in many ophthalmic medications can worsen ocular surface inflammation. ${ }^{5}$ For patients who require artificial tears more than four to six times per day, preservative-free artificial tears must be used. ${ }^{5}$

\section{Resources for physicians}

- The Canadian Ophthalmological Society (www.cos-sco.ca)

- American Association of Ophthalmology (www.aao.org)

- American Optometric Association (www.aoa.org)

\section{References}

1. Lemp MA. Advances in understanding and managing dry eye disease. Am J Ophthalmol 2008;146: 350-6.

2. Buchholz P, Steeds CS, Stern LS, et al. Utility assessment to measure the impact of dry eye disease. Ocul Surf 2006;4:155-61.

3. American Academy of Ophthalmology Cornea /External Disease Panel. Dry eye syndrome. Limited revision. San Francisco (CA): American Academy of Ophthalmology; 2011.

4. Noble J, Lloyd JC. The red eye. CMAJ 2011;183:81.

5. Management and therapy of dry eye disease: report of the Management and Therapy Subcommittee of the International Dry Eye WorkShop. Ocul Surf 2007;5:163-78.
Patients at risk of vision loss require prompt referral to an ophthalmologist

Referral to an ophthalmologist should occur in cases of severe pain or visual loss. ${ }^{1,4}$ Visual acuity should be assessed before referral. Referral is also recommended for patients whose symptoms do not respond to first-line therapy and those with a history of trauma, chemical injury, Bell palsy, autoimmune disease, (e.g., Sjögren syndrome) or other ocular surface conditions (e.g., herpes simplex, herpes zoster ophthalmicus). ${ }^{3,4}$ Ophthalmologists may use topical cyclosporine, scleral contact lenses, punctal occlusion or tarsorrhaphy to treat severe cases of dry eye. ${ }^{5}$

Competing interests: None declared.

This article has been peer reviewed.

Affiliations: Department of Ophthalmology (Mather), Schulich School of Medicine and Dentistry (Sharma), London, Ont.; Ivey Eye Institute (Mather), London, Ont.

Correspondence to: Rahul Sharma, rsharma2014 @meds.uwo.ca

CMAJ 2014. DOI:10.1503/cmaj.131590 